Mathematical Modelling AND ANALysis

Volume 18 Number 3, June 2013, 415-431

http://dx.doi.org/10.3846/13926292.2013.805172

(c) Vilnius Gediminas Technical University, 2013
Publisher: Taylor\&Francis and VGTU

http://www.tandfonline.com/TMMA

Print ISSN: 1392-6292

Online ISSN: 1648-3510

\title{
Analysis of Stabilized Finite Volume Method for Poisson Equation*
}

\author{
Tong Zhang ${ }^{a, b}$, Pengzhan Huang ${ }^{c}$ and Shunwei Xu ${ }^{a}$ \\ ${ }^{a}$ Henan Polytechnic University \\ 454003 Jiaozuo, China \\ ${ }^{b}$ Universidade Federal do Paraná \\ 81531-990 Curitiba, Brazil \\ ${ }^{c}$ Xinjiang University \\ 830046 Urumqi, China \\ E-mail(corresp.): zhangtong0616@163.com \\ E-mail: hpzh007@yahoo.cn \\ E-mail: xushunwei@hpu.edu.cn
}

Received August 25, 2012; revised May 8, 2013; published online June 1, 2013

\begin{abstract}
In this work, we systematically analyze a stabilized finite volume method for the Poisson equation. On stating the convergence of this method, optimal error estimates in different norms are obtained by establishing the adequate connections between the finite element and finite volume methods. Furthermore, some superconvergence results are established by using $L^{2}$-projection method on a coarse mesh based on some regularity assumptions for Poisson equation. Finally, some numerical experiments are presented to confirm the theoretical findings.
\end{abstract}

Keywords: Poisson equation, finite volume method, projection method, superconvergence.

AMS Subject Classification: 65M08; 65N12; 35J05.

\section{Introduction}

Finite volume method (FVM) as one of important numerical discretization techniques has been widely employed to solve the fluid dynamics problems [8]. The basic idea of FVM is to approximate discrete fluxes of a partial differential equation using a finite element procedure based on volumes or control volumes, so FVM is also known as covolume methods, or box methods [1], marker and cell methods [5], generalized difference methods [17]. The difficulty in the

\footnotetext{
* This work was supported by the NSF of China (Nos. 11126117, 11226319), Doctoral Foundation of Henan Polytechnic University (B2012-098), the Natural Science Foundation of Xinjiang Province (No. 2013211B01), the Doctoral Foundation of Xinjiang University (No. BS120102). The first author was partially supported by the CAPES and CNPq, Brazil.
} 
analysis of finite volume method arises from trial and test functions to lie in different spaces and to be associated with different meshes. This can be resolved by introducing a transfer map which allows to rewrite the finite element formulation as its classical finite-volume-like counterpart, i.e., using piecewise constant test functions. We can refer to $[7,10,14,21]$ and the references therein for more recent developments about finite volume method.

The mixed finite element method has became a popular method for solving the partial differential equations arising in solid and fluid mechanics [4]. Its popularity is due to the fact that in some cases a vector variable is the primary variable in which one is interested. The mixed method is developed to approximate both this variable and a scalar variable simultaneously and to give accurate approximations of both variables. The mixed finite element formulations use two different approximate spaces. These two spaces must be chosen carefully to satisfy the inf-sup stability condition. There exist rich choices for these special spaces for the equations of solid and fluid mechanics (see $[4,19]$ and the references therein).

Much attention has recently been attracted to use the $P_{1}-P_{1}$ pair for the fluid mechanics equations, particularly for the Stokes and Navier-Stokes equations (see $[2,9,12]$ ). Although this pair does not satisfy the inf-sup stability condition, it offers simple and practical uniform data structure and adequate accuracy. Many stabilization techniques have been proposed to stabilize the unstable pairs such as penalty method, pressure projection, velocity correction and residual stabilization methods. Among these methods, the pressure projection stabilization method [2] is a preferable choice in that it is free of stabilization parameters, does not require any calculation of high-order derivations or edge-based data structure, and can be implemented at the element level. Recent studies have been focused on stabilization of the $P_{1}-P_{1}$ or $P_{1}-P_{0}$ using this type of stabilization for the Stokes and Navier-Stokes equations, see $[10,11,13,16,22]$ and the references therein.

There have been some attempts to use the velocity projection stabilization method for solving Poisson equation. Preliminary computational studies were given, and numerical results were reported using the low-order finite element pairs (see [23]). However, the analysis of finite volume method is still lacking for the second order equations. The analysis of the mixed finite volume method for Poisson equations is much more dedicate than for the Stokes equations since the latter equations are naturally given in mixed form. The pair $H^{1}(\Omega)^{2} \times L^{2}(\Omega)$ is used for the Stokes equations while we can relax the regularity of the exact solution for Poisson equation such that this set is still valid. In this work, we provide a systematical finite volume analysis of the velocity projection stabilization method for Poisson equation. Stability results for the finite volume solution are presented. Optimal error estimates for velocity and pressure are obtained. Furthermore, some superconvergence results are established for Poisson equation by using a $L^{2}$-projection on the coarse mesh. Like other results in the family of $L^{2}$-projection methods (see $[5,6,15]$ ), the superconvergence results presented in this work are based on some regularity assumptions for Poisson problem on the quasi-uniform triangulation partitions. Different from [18], this paper considers the mixed finite volume method for Poisson 
equations. We not only provide the stability and optimal error estimates of numerical solution, but also present some meaningful superconvergence results by using the $L^{2}$-projections between different finite element spaces.

The rest of this paper is organized as follows. In Section 2, some results about Poisson equation and its mixed finite element scheme are recalled. Section 3 is devoted to establish the stabilized finite volume formulation for Poisson problem. Optimal error estimates are derived in Section 4. In Section 5, some superconvergence results are developed by introducing the $L^{2}$-projection methods between different finite element spaces. In Section 6 , some numerical experiments are provided to verify the established theoretical findings. Finally, we end with a short conclusion in Section 7.

\section{Preliminaries}

Let $\Omega$ be a bounded domain in $\mathbb{R}^{2}$ assumed to have a Lipschitz continuous polygonal boundary $\partial \Omega$. We consider the following Poisson equation

$$
\begin{cases}-\Delta p=f & \text { in } \Omega \\ p=0 & \text { on } \partial \Omega\end{cases}
$$

This equation arises from many physical and mechanical phenomena, for example, the equilibrium of an elastic membrane fixed on the boundary and subject to a load of intensity $f$, the equilibrium of some quantity like chemical or temperature distribution when $f=0$.

Standard definitions are used for the Sobolev spaces $W^{m, r}(\Omega)$, with the norm $\|\cdot\|_{m, r}$ and the seminorm $|\cdot|_{m, r}, m, r \geq 0$. We will write $H^{m}(\Omega)$ for $W^{m, 2}(\Omega)$ and $\|\cdot\|_{m}$ for $\|\cdot\|_{m, 2}$. The notation $(\cdot, \cdot)$ indicates the inner product on the domain $\Omega$.

In order to state a mixed formulation for (2.1), we define the spaces

$$
V=H(\operatorname{div} ; \Omega)^{2}, \quad X=H_{0}^{1}(\Omega), \quad W=L^{2}(\Omega), \quad W_{1}=L^{2}(\Omega)^{2} .
$$

Letting $u=-\nabla p$, the mixed formulation of equation (2.1) is to find $u \in$ $V, p \in W$, such that

$$
\begin{cases}(u, v)-(\nabla \cdot v, p)=0 & \forall v \in V, \\ (\nabla \cdot u, w)=(f, w) & \forall w \in W .\end{cases}
$$

In fact, we do not need $u \in V$, we only require $u \in W_{1}$. Denoting

$$
a(u, v)=(u, v), \quad d(v, p)=-(v, \nabla p) .
$$

Then, applying the Green formula, we have the following variational formulation for equation (2.1)

$$
a(u, v)-d(v, p)+d(u, w)=(f, w) \quad \forall(u, p),(v, w) \in W_{1} \times X .
$$

The following lemma establishes the existence, uniqueness of the solution to Poisson equation (2.2) under some weak regularities. 
Lemma 1. The bilinear forms $d(\cdot, \cdot)$ satisfies the $L B B$ condition, i.e., there exists a constant $\beta_{1}>0$, such that

$$
\inf _{w \in X} \sup _{v \in W_{1}} \frac{d(v, w)}{\|v\|_{W_{1}}\|w\|_{X}} \geq \beta_{1} .
$$

Proof. See Lemma 2.2 of Ref. [18].

Theorem 1. There exists a unique solution $(u, p) \in W_{1} \times X$ to problem (2.2).

Proof. Combining Lemma 1 and Lax-Milgram Theorem yields the desired result immediately.

Let $\mathcal{T}_{h}=\{K\}$ be a regular, quasi-uniform partition of the domain $\Omega$ into a finite number of triangulations, $h_{K}=\operatorname{diam}(K), h=\max \left\{h_{K}: K \in \mathcal{T}_{h}\right\}$. We consider the following mixed finite element spaces

$$
\begin{aligned}
& W_{h}=\left\{v \in C^{0}(\bar{\Omega})^{2} \cap W_{1}:\left.v\right|_{K} \in P_{1}(K)^{2} \forall K \in \mathcal{T}_{h}\right\}, \\
& X_{h}=\left\{w \in C^{0}(\bar{\Omega}) \cap X:\left.w\right|_{K} \in P_{1}(K) \forall K \in \mathcal{T}_{h}\right\},
\end{aligned}
$$

where $P_{1}(K)$ is the set of linear polynomials on $K$.

Set $I_{h}$ and $J_{h}$ be two interpolation operators from $W_{1}$ and $X$ into $W_{h}$ and $X_{h}$, respectively, such that for any $v \in H^{1}(\Omega)^{2}$ and $w \in H^{2}(\Omega)$ (see [4])

$$
\left\|v-I_{h} v\right\|_{0} \leq C h\|v\|_{1}, \quad\left\|w-J_{h} w\right\|_{i} \leq C_{2} h^{2-i}\|w\|_{2}, \quad i=0,1 .
$$

Obviously, the lowest equal-order finite element pair does not satisfy the discrete inf-sup condition (see [19])

$$
\beta\left\|v_{h}\right\|_{0} \leq \sup _{0 \neq w_{h} \in X_{h}} \frac{\left(v_{h}, \nabla w_{h}\right)}{\left\|\nabla w_{h}\right\|_{0}} \quad \forall v_{h} \in W_{h}
$$

where the constant $\beta>0$ is independent of $h$. In order to overcome the restriction (2.4), we define a $L^{2}$-projection operator $\Pi_{h}: W_{1} \rightarrow P_{0}^{2}$ by

$$
\left(u, v_{h}\right)=\left(\Pi_{h} u, v_{h}\right), \quad u \in W_{1}, v_{h} \in P_{0}^{2},
$$

where $P_{0}^{2}$ is the 2-D piecewise constant space associated with the triangles $\mathcal{T}_{h}$. And assume $\Pi_{h}$ satisfies the following properties (see [3]):

$$
\begin{gathered}
\left\|\Pi_{h} u\right\|_{0} \leq C\|u\|_{0} \quad \forall u \in W_{1} ; \\
\left\|u-\Pi_{h} u\right\|_{0} \leq C h\|u\|_{1} \quad \forall u \in H^{1}(\Omega)^{2} .
\end{gathered}
$$

With the help of $(2.5)$, we can define a bilinear form $G_{h}(\cdot, \cdot)$ by

$$
\begin{aligned}
G_{h}\left(u_{h}, v_{h}\right) & =\left(u_{h}-\Pi_{h} u_{h}, v_{h}\right) \\
& =\left(u_{h}-\Pi_{h} u_{h}, v_{h}-\Pi_{h} v_{h}\right) \quad \forall u_{h}, v_{h} \in W_{h} .
\end{aligned}
$$

Remark. The bilinear form $G_{h}(\cdot, \cdot)$ in $(2.7)$ is symmetric, semi-positive definite form generated on each local element $K$. 
The finite element discrete scheme for (2.1) on $W_{h} \times X_{h}$ can be defined as

$$
\begin{aligned}
& a\left(u_{h}^{e}, v_{h}\right)-d\left(v_{h}, p_{h}^{e}\right)+d\left(u_{h}^{e}, w_{h}\right)+G_{h}\left(u_{h}^{e}, v_{h}\right)=\left(f, w_{h}\right) \\
& \quad \forall\left(v_{h}, w_{h}\right) \in W_{h} \times X_{h} .
\end{aligned}
$$

Denoting the generalized bilinear form

$$
B_{h}\left(\left(u_{h}^{e}, p_{h}^{e}\right),\left(v_{h}, w_{h}\right)\right)=a\left(u_{h}^{e}, v_{h}\right)-d\left(v_{h}, p_{h}^{e}\right)+d\left(u_{h}^{e}, w_{h}\right)+G_{h}\left(u_{h}^{e}, v_{h}\right) .
$$

It has been shown that this general bilinear form (2.9) satisfies the continuity and weak coercivity properties (see [18]):

$$
\begin{gathered}
B_{h}\left(\left(u_{h}^{e}, p_{h}^{e}\right) ;\left(v_{h}, w_{h}\right)\right) \leq C\left(\left\|u_{h}^{e}\right\|_{0}+\left\|\nabla p_{h}^{e}\right\|_{0}\right)\left(\left\|v_{h}\right\|_{0}+\left\|\nabla w_{h}\right\|_{0}\right), \\
\sup _{0 \neq\left(v_{h}, w_{h}\right) \in W_{h} \times X_{h}} \frac{B_{h}\left(\left(u_{h}^{e}, p_{h}^{e}\right) ;\left(v_{h}, w_{h}\right)\right)}{\left\|v_{h}\right\|_{0}+\left\|\nabla w_{h}\right\|_{0}} \geq \beta\left(\left\|u_{h}^{e}\right\|_{0}+\left\|\nabla p_{h}^{e}\right\|_{0}\right),
\end{gathered}
$$

where $\beta>0$ is a constant, independent of mesh parameter $h$.

Thanks to (2.9), we can simplify the equation (2.8) into the following mixed finite element formulation: Find $\left(u_{h}^{e}, p_{h}^{e}\right) \in W_{h} \times X_{h}$ such that

$$
B_{h}\left(\left(u_{h}^{e}, p_{h}^{e}\right),\left(v_{h}, w_{h}\right)\right)=\left(f, w_{h}\right) \quad \forall\left(v_{h}, w_{h}\right) \in W_{h} \times X_{h} .
$$

Combining the classical saddle theorem and (2.10)-(2.11), we know that the system (2.12) admits a unique solution. Moreover, the following error estimates for the finite element solution $\left(u_{h}^{e}, p_{h}^{e}\right)$ hold (see [18])

$$
\begin{aligned}
& \left\|p-p_{h}^{e}\right\|_{0}+h\left(\left\|\nabla\left(p-p_{h}^{e}\right)\right\|_{0}+\left\|u-u_{h}^{e}\right\|_{0}\right) \\
& \quad \leq C h^{2}\left(\|u\|_{1}+\|p\|_{2}+\|f\|_{0}\right) .
\end{aligned}
$$

\section{$3 \quad$ Stabilized Finite Volume Method}

This section is devoted to present the stabilized finite volume formulation for Poisson equation and establish the existence uniqueness for the approximation solution.

Let $\mathcal{P}$ be the set containing all the interior nodes associated with the triangulations $K, \mathcal{N}_{h}$ denotes the set of all nodes $\mathcal{T}_{h}$. Based on the partition $\mathcal{T}_{h}$, we introduce the corresponding dual partition $\mathcal{T}_{h}^{*}$. Here, we choose the barycenter $Q$ of a element $K \in \mathcal{T}_{h}$, and the midpoints $M$ on the edges of $K$, then connect $Q$ to $M$ by straight line. For an arbitrary vertex $x_{i} \in K$, let $\widetilde{K}_{i}$ be the polygonal which is called a control volume. Then, we have $\bar{\Omega}=\bigcup_{x_{i}, i \in \mathcal{N}_{h}} \widetilde{K}_{i}$, the dual mesh $\mathcal{T}_{h}^{*}$ is the set of these control volumes.

The dual finite element space is defined as

$$
\widetilde{X}_{h}=\left\{\widetilde{v} \in W: \widetilde{v} \in P_{0}\left(\widetilde{K}_{i}\right) \forall \widetilde{K}_{i} \in \mathcal{T}_{h}^{*} ;\left.\widetilde{v}\right|_{\partial \widetilde{K}_{i}}=0\right\} .
$$

It is clearly that the dimensions of $X_{h}$ and $\widetilde{X}_{h}$ are the same. Furthermore, there exists an invertible linear mapping $\Gamma_{h}: X_{h} \rightarrow \widetilde{X}_{h}$ such that

$$
\Gamma_{h} v_{h}(x)=\sum_{i=1}^{\mathcal{N}_{h}} v_{h}\left(x_{i}\right) \phi_{i}(x), \quad x \in \Omega, v_{h} \in X_{h},
$$


where $\phi_{i}(x)$ is the basis functions associated with the dual partition $\mathcal{T}_{h}^{*}$ :

$$
\phi_{i}(x)= \begin{cases}1, & x \in \widetilde{K}_{i} \\ 0, & \text { otherwise }\end{cases}
$$

The above idea of connecting the different spaces through the mapping $\Gamma_{h}$ was introduced by Li in [17] for the elliptic problem. Furthermore, the mapping $\Gamma_{h}$ has the following properties (see [21]).

Lemma 2. Let $K \in \mathcal{T}_{h}$, if $v_{h} \in X_{h}$ and $1 \leq r \leq \infty$, then

$$
\int_{K}\left(v_{h}-\Gamma_{h} v_{h}\right) d x=0, \quad\left\|v_{h}-\Gamma_{h} v_{h}\right\|_{L^{r}(K)^{d}} \leq C h_{K}\left\|v_{h}\right\|_{W^{1, r}(K)^{d}},
$$

where $h_{K}$ is the diameter of the element $K$.

Applying Green's formula, we present the finite volume approximation to (2.1) as finding $u_{h} \in W_{h}, p_{h} \in X_{h}$, such that for $\forall\left(v_{h}, w_{h}\right) \in W_{h} \times X_{h}$

$$
a\left(u_{h}, v_{h}\right)-d\left(v_{h}, p_{h}\right)+D\left(u_{h}, w_{h}\right)+G_{h}\left(u_{h}, v_{h}\right)=\left(f, \Gamma_{h} w_{h}\right),
$$

where

$$
D\left(u_{h}, w_{h}\right)=\sum_{j=1}^{\mathcal{N}_{h}} w_{h}\left(\mathcal{P}_{j}\right) \int_{\partial \widetilde{K}_{j}} u_{h} \cdot n d x, \quad u_{h} \in W_{h}, w_{h} \in X_{h},
$$

where $n$ is the unit outward to $\partial \widetilde{K}_{j}$. Setting

$$
C_{h}\left(\left(u_{h}, p_{h}\right),\left(v_{h}, w_{h}\right)\right)=a\left(u_{h}, v_{h}\right)-d\left(v_{h}, p_{h}\right)+D\left(u_{h}, w_{h}\right)+G_{h}\left(u_{h}, v_{h}\right),
$$

then, system (3.1) can be rewritten as

$$
C_{h}\left(\left(u_{h}, p_{h}\right),\left(v_{h}, w_{h}\right)\right)=\left(f, \Gamma_{h} w_{h}\right) \quad \forall\left(v_{h}, w_{h}\right) \in W_{h} \times X_{h} .
$$

The next lemma establishes the relationship between the finite element and finite volume methods for Poisson equation.

Lemma 3. It holds that

$$
D\left(u_{h}, w_{h}\right)=-\left(u_{h}, \nabla w_{h}\right) \quad \forall\left(u_{h}, w_{h}\right) \in W_{h} \times X_{h} .
$$

Proof. From the definition of $D(\cdot, \cdot)$, and combining Lemma 2, Green's formula with the fact that $\operatorname{div} u_{h}$ is a constant that

$$
\begin{aligned}
D\left(u_{h}, w_{h}\right) & =\sum_{i=1}^{\mathcal{N}_{h}} \int_{\widetilde{K}_{i}} \operatorname{div} u_{h} \cdot \Gamma_{h} w_{h} d x=\sum_{i=1}^{\mathcal{N}_{h}} \int_{K \cap \widetilde{K}_{i}} \operatorname{div} u_{h} \cdot w_{h} d x \\
& =-\sum_{K \in \mathcal{T}_{h}} \int_{K} u_{h} \cdot \nabla w_{h} d x=-\left(u_{h}, \nabla w_{h}\right) .
\end{aligned}
$$

Now, we are in the position of establishing the continuity and weak coercivity for the general bilinear form $C_{h}\left(\left(u_{h}, p_{h}\right),\left(v_{h}, w_{h}\right)\right)$. 
Lemma 4. It holds that for all $\left(u_{h}, p_{h}\right),\left(v_{h}, w_{h}\right) \in W_{h} \times X_{h}$

$$
\left|C_{h}\left(\left(u_{h}, p_{h}\right),\left(v_{h}, w_{h}\right)\right)\right| \leq C\left(\left\|u_{h}\right\|_{0}+\left\|\nabla p_{h}\right\|_{0}\right)\left(\left\|v_{h}\right\|_{0}+\left\|\nabla w_{h}\right\|_{0}\right) .
$$

Moreover,

$$
\beta_{2}\left(\left\|u_{h}\right\|_{0}+\left\|\nabla p_{h}\right\|_{0}\right) \leq \sup _{0 \neq\left(v_{h}, w_{h}\right) \in\left(W_{h}, X_{h}\right)} \frac{\left|C_{h}\left(\left(u_{h}, p_{h}\right) ;\left(v_{h}, w_{h}\right)\right)\right|}{\left\|v_{h}\right\|_{0}+\left\|\nabla w_{h}\right\|_{0}},
$$

where the constant $\beta_{2}>0$ is independent of $h$.

Proof. The continuity of (3.3) can easily be shown using the definition of the bilinear form $C_{h}((\cdot, \cdot),(\cdot, \cdot))$ and Lemma 3.

In the following, we will prove the weak coercivity (3.4). For any fixed $p_{h} \in X_{h}$, there exists a unique $\phi \in W_{1}$, satisfying $-\left(\phi, \nabla p_{h}\right)=\left\|\nabla p_{h}\right\|_{0}^{2}$ and $\|\phi\|_{0} \leq C\left\|\nabla p_{h}\right\|_{0}$. Let $\phi_{h}=I_{h} \phi$, where $I_{h}$ is the interpolation operator from $W_{1}$ to $W_{h}$, satisfies $(2.3)$, and possesses the following property:

$$
\left(\phi-I_{h} \phi, w_{h}\right)=0 \quad \forall w_{h} \in W_{h} .
$$

Combining the definition of $I_{h}$ in $(3.5)$ with the fact that $\nabla p_{h}$ is piecewise constant, we arrive at

$$
-\left(\phi_{h}, \nabla p_{h}\right)=-\left(\phi, \nabla p_{h}\right)=\left\|\nabla p_{h}\right\|_{0}^{2} \quad \forall p_{h} \in X_{h} .
$$

By using Lemma 2, (3.5), (3.6) and Cauchy inequality yields

$$
\begin{aligned}
C_{h}\left(\left(u_{h}, p_{h}\right),\left(\phi_{h}, 0\right)\right) & =\left(u_{h}, \phi_{h}\right)+\left(\phi_{h}, \nabla p_{h}\right)+G_{h}\left(u_{h}, \phi_{h}\right) \\
& \leq\left\|u_{h}\right\|_{0}\left\|\phi_{h}\right\|_{0}+\left(\phi, \nabla p_{h}\right)+C\left\|u_{h}\right\|_{0}\left\|\phi_{h}\right\|_{0} \\
& \leq\left\|u_{h}\right\|_{0}\left\|\nabla p_{h}\right\|_{0}-\left\|\nabla p_{h}\right\|_{0}^{2}+C\left\|u_{h}\right\|_{0}\left\|\nabla p_{h}\right\|_{0} \\
& \leq\left(1+C^{2}\right)\left\|u_{h}\right\|_{0}^{2}-\frac{1}{2}\left\|\nabla p_{h}\right\|_{0}^{2} .
\end{aligned}
$$

Setting $\left(v_{h}, q_{h}\right)=\left(u_{h}-\alpha \phi_{h}, p_{h}\right)$ with $\alpha>0$, applying (3.7) and Lemma 3 yields

$$
\begin{aligned}
& C_{h}\left(\left(u_{h}, p_{h}\right),\left(u_{h}-\alpha \phi_{h}, p_{h}\right)\right) \\
& \quad=C_{h}\left(\left(u_{h}, p_{h}\right),\left(u_{h}, p_{h}\right)\right)-\alpha C_{h}\left(\left(u_{h}, p_{h}\right),\left(\phi_{h}, 0\right)\right) \\
& \quad=\left(u_{h}, u_{h}\right)+G_{h}\left(u_{h}, u_{h}\right)-\alpha\left(1+C^{2}\right)\left\|u_{h}\right\|_{0}^{2}+\frac{\alpha}{2}\left\|\nabla p_{h}\right\|_{0}^{2} \\
& \quad \geq\left(1-\alpha\left(1+C^{2}\right)\right)\left\|u_{h}\right\|_{0}^{2}+\frac{\alpha}{2}\left\|\nabla p_{h}\right\|_{0}^{2} .
\end{aligned}
$$

Choosing $\alpha=\frac{1}{2\left(1+C^{2}\right)}$ we have

$$
C_{h}\left(\left(u_{h}, p_{h}\right),\left(v_{h}, w_{h}\right)\right) \geq C\left(\left\|u_{h}\right\|_{0}+\left\|\nabla p_{h}\right\|_{0}\right)^{2} .
$$

On the other hand,

$$
\begin{aligned}
\left\|u_{h}-\alpha \phi_{h}\right\|_{0}+\left\|\nabla p_{h}\right\|_{0} & \leq\left(\left\|u_{h}\right\|+\alpha\left\|\phi_{h}\right\|_{0}+\left\|\nabla p_{h}\right\|_{0}\right) \\
& \leq C\left(\left\|u_{h}\right\|+\left\|\nabla p_{h}\right\|_{0}\right) .
\end{aligned}
$$


Combining (3.8) with (3.9), we have finished the proof.

It follows from Lemma 4 that system (3.1) has a unique solution, and satisfies

$$
\left\|u_{h}\right\|_{0}+\left\|\nabla p_{h}\right\|_{0} \leq C\|f\|_{0}
$$

\section{Error Estimates}

In this section, we present some optimal error estimates of the stabilized finite volume solutions for equation (3.1) under some regularities about $f$.

Theorem 2. Let $(u, p)$ and $\left(u_{h}, p_{h}\right)$ be the solutions of (2.1) and (3.1), respectively. Then we have

$$
\left\|\nabla\left(p-p_{h}\right)\right\|_{0}+\left\|u-u_{h}\right\|_{0} \leq C h\left(\|p\|_{2}+\|u\|_{1}+\|f\|_{0}\right) .
$$

Proof. Subtracting (2.12) from (3.2) and using Lemma 3 gives

$$
C_{h}\left(\left(u_{h}-u_{h}^{e}, p_{h}-p_{h}^{e}\right),\left(v_{h}, w_{h}\right)\right)=\left(f, \Gamma_{h} w_{h}-w_{h}\right) .
$$

Obviously, we deduce from Lemmas 2 and 4 that

$$
\begin{gathered}
\sup _{\left(v_{h}, w_{h}\right) \in\left(W_{h}, X_{h}\right)} \frac{C_{h}\left(\left(u_{h}-u_{h}^{e}, p_{h}-p_{h}^{e}\right),\left(v_{h}, w_{h}\right)\right)}{\left\|v_{h}\right\|_{0}+\left\|\nabla w_{h}\right\|_{0}} \\
\geq \beta_{2}\left(\left\|u_{h}-u_{h}^{e}\right\|_{0}+\left\|\nabla\left(p_{h}-p_{h}^{e}\right)\right\|_{0}\right), \\
\left|\left(f, \Gamma_{h} w_{h}-w_{h}\right)\right| \leq\|f\|_{0}\left\|\Gamma_{h} w_{h}-w_{h}\right\|_{0} \leq C h\|f\|_{0}\left\|\nabla w_{h}\right\|_{0} .
\end{gathered}
$$

Combining above equations with (4.1), we arrive at

$$
\left\|u_{h}-u_{h}^{e}\right\|_{0}+\left\|\nabla\left(p_{h}-p_{h}^{e}\right)\right\|_{0} \leq C h\|f\|_{0},
$$

which, together with (2.13), we have finished the proof.

To obtain the estimate of the error $p-p_{h}$ in $L^{2}$-norm, we use the duality argument. Considering the following problem by seeking $(\phi, \varphi) \in W_{1} \times X$ such that

$$
(v, \phi)+d(v, \varphi)-d(\phi, w)=\left(p-p_{h}, w\right) \quad \forall(v, w) \in W_{1} \times X .
$$

When $\Omega$ is convex, the solutions of (4.2) satisfy

$$
\|\phi\|_{1}+\|\varphi\|_{2} \leq C\left\|p-p_{h}\right\|_{0}
$$

Theorem 3. Let $(u, p)$ and $\left(u_{h}, p_{h}\right)$ be the solutions of (2.1) and (3.1), respectively, and $f \in H^{1}(\Omega)$. Then

$$
\left\|p-p_{h}\right\|_{0} \leq C h^{2}\left(\|p\|_{2}+\|u\|_{1}+\|f\|_{1}\right) .
$$


Proof. Choosing $(v, w)=\left(u-u_{h}, p-p_{h}\right)$ in $(4.2)$, letting $(v, w)=\left(I_{h} v, J_{h} w\right)$ in $(2.2),\left(v_{h}, w_{h}\right)=\left(I_{h} v, J_{h} w\right)$ in $(3.2)$, where $I_{h}, J_{h}$ are two interpolation operators and satisfy $(2.3)$, we arrive at

$$
\begin{aligned}
\left\|p-p_{h}\right\|_{0}^{2}= & C_{h}\left(\left(u-u_{h}, p-p_{h}\right),\left(\phi-I_{h} \phi, \varphi-J_{h} \varphi\right)\right)+G_{h}\left(u, I_{h} \phi\right) \\
& +\left(f, J_{h} \varphi-\Gamma_{h} J_{h} \varphi\right)-G_{h}\left(\phi, u-u_{h}\right) .
\end{aligned}
$$

It follows from (2.5)-(2.7), Theorem 2 and (4.3) that

$$
\begin{aligned}
& \left|G_{h}\left(u, I_{h} \phi\right)\right| \leq C h^{2}\|u\|_{1}\|\phi\|_{1} \leq C h^{2}\|u\|_{1}\left\|p-p_{h}\right\|_{0}, \\
& \left|G_{h}\left(u-u_{h}, \phi\right)\right| \leq C h\left\|u-u_{h}\right\|_{0}\|\phi\|_{1} \leq C h^{2}\left\|p-p_{h}\right\|_{0}\left(\|p\|_{2}+\|u\|_{1}+\|f\|_{0}\right), \\
& \quad\left|C_{h}\left(\left(u-u_{h}, p-p_{h}\right),\left(\phi-I_{h} \phi, \varphi-J_{h} \varphi\right)\right)\right| \\
& \quad \leq\left(\left\|u-u_{h}\right\|_{0}+\left\|\nabla\left(p-p_{h}\right)\right\|_{0}\right)\left(\left\|\phi-I_{h} \phi\right\|_{0}+\left\|\nabla\left(\varphi-J_{h} \varphi\right)\right\|_{0}\right) \\
& \quad \leq C h^{2}\left(\|\phi\|_{1}+\|\varphi\|_{2}\right)\left(\|p\|_{2}+\|u\|_{1}+\|f\|_{0}\right) \\
& \quad \leq C h^{2}\left\|p-p_{h}\right\|_{0}\left(\|p\|_{2}+\|u\|_{1}+\|f\|_{0}\right) .
\end{aligned}
$$

In addition, let $P_{h} f$ be defined by

$$
\left.P_{h} f\right|_{K}=\frac{1}{|K|} \int_{K} f(x) d x \quad K \in \mathcal{T}_{h},
$$

where $|K|$ is the area of the element $K$. Then, it follows from (2.5), (4.3) and Lemma 2 that

$$
\begin{aligned}
\left|\left(f, J_{h} \varphi-\Gamma_{h} J_{h} \varphi\right)\right| & =\left|\left(f-P_{h} f, J_{h} \varphi-\Gamma_{h} J_{h} \varphi\right)\right| \\
& \leq\left\|f-P_{h} f\right\|_{0}\left\|J_{h} \varphi-\Gamma_{h} J_{h} \varphi\right\|_{0} \\
& \leq C h^{2}\|f\|_{1}\left\|\nabla J_{h} \varphi\right\|_{0} \leq C h^{2}\|f\|_{1}\left\|p-p_{h}\right\|_{0} .
\end{aligned}
$$

Combining above inequalities with (4.4) yields the desired result.

\section{Superconvergence}

The post-processing technique introduced by Wang $[15,20]$ is onto project the numerical solution to another finite element space with different mesh size. The difference in these mesh sizes can be used to achieve a superconvergence after the post-processing procedure. Let $\mathcal{T}_{\tau_{i}}(i=1,2)$ be another two finite element partitions with mesh sizes $\tau_{i}$, where $h \ll \tau_{i}(i=1,2)$. Assume that $\tau_{i}(i=1,2)$ and $h$ have the following relation

$$
\tau_{i}=h^{\alpha_{i}}
$$

with $\alpha_{1}, \alpha_{2} \in(0,1), \alpha_{i}$ play an important role in achieving the superconvergence for the stabilized finite volume solution $\left(u_{h}, p_{h}\right)$. Let $V_{\tau_{1}}$ and $P_{\tau_{2}}$ be two finite element spaces consisting of piecewise polynomials of degree $r$ and $t$, respectively, associated with the partition $\mathcal{T}_{\tau_{i}}$. We define two $L^{2}$ projections $Q_{\tau_{1}}$ and $R_{\tau_{2}}$ from $L^{2}(\Omega)$ onto the finite element spaces $V_{\tau_{1}}$ and $P_{\tau_{2}}$, respectively. In the following, we will analyze the errors of $p-Q_{\tau_{1}} p_{h}$ and $u-R_{\tau_{2}} u_{h}$.

The following two lemmas provide the error estimates for $Q_{\tau_{1}} p-Q_{\tau_{1}} p_{h}$ and $R_{\tau_{2}} u-R_{\tau_{2}} u_{h}$, respectively. 
Lemma 5. Assume $V_{\tau_{1}} \in L^{2}(\Omega)$ and $f \in H^{1}(\Omega)$, then there is a constant $C$, independent of $h$ and $\tau_{1}$ such that

$$
\left\|Q_{\tau_{1}} p-Q_{\tau_{1}} p_{h}\right\|_{0} \leq C h^{2}\left(\|u\|_{1}+\|p\|_{2}+\|f\|_{1}\right) .
$$

Proof. From the definition of $\|\cdot\|_{0}$ and $Q_{\tau_{1}}$, we have

$$
\begin{aligned}
& \left\|Q_{\tau_{1}} p-Q_{\tau_{1}} p_{h}\right\|_{0}=\sup _{\phi \in L^{2}(\Omega),\|\phi\|_{0}=1}\left|\left(Q_{\tau_{1}} p-Q_{\tau_{1}} p_{h}, \phi\right)\right| \\
& \left(Q_{\tau_{1}} p-Q_{\tau_{1}} p_{h}, \phi\right)=\left(p-p_{h}, Q_{\tau_{1}} \phi\right) .
\end{aligned}
$$

Then we obtain that

$$
\left\|Q_{\tau_{1}} p-Q_{\tau_{1}} p_{h}\right\|_{0}=\sup _{\phi \in L^{2}(\Omega),\|\phi\|_{0}=1}\left|\left(p-p_{h}, Q_{\tau_{1}} \phi\right)\right| .
$$

Considering the following problem

$$
\left(u-u_{h}, v\right)-d\left(v, p-p_{h}\right)+d\left(u-u_{h}, w\right)=\left(p-p_{h}, Q_{\tau_{1}} \phi\right) .
$$

Because of the convexity of domain $\Omega$, problem (5.2) has a unique solution and satisfies

$$
\|w\|_{2}+\|v\|_{1} \leq C\left\|Q_{\tau_{1}} \phi\right\|_{0} .
$$

Let $\left(v_{h}, w_{h}\right) \in W_{h} \times X_{h}$ be the usual piecewise linear interpolant of $(v, w)$, which satisfies (2.3). From the continuous equation (2.1), we have the following finite volume variational formulation

$$
\left(u, v_{h}\right)+\left(\nabla p, v_{h}\right)+\left(\nabla \cdot u, \Gamma_{h} w_{h}\right)=\left(f, \Gamma_{h} w_{h}\right) .
$$

The following error equation can be obtained from (3.2) and (5.4)

$$
C_{h}\left(\left(u-u_{h}, p-p_{h}\right),\left(v_{h}, w_{h}\right)\right)+G_{h}\left(u, v_{h}\right)=0 \quad \forall\left(v_{h}, w_{h}\right) \in W_{h} \times X_{h} .
$$

Combining equations $(5.2),(5.5)$ with Lemma 2 , and the fact that $\nabla \cdot u_{h}$ is a constant yields

$$
\begin{aligned}
\left(Q_{\tau_{1}} \phi, p-p_{h}\right)= & \left(u-u_{h}, v-v_{h}\right)-d\left(v-v_{h}, p-p_{h}\right)+d\left(u-u_{h}, w-w_{h}\right) \\
& +\left(\nabla \cdot\left(u-u_{h}\right), w_{h}-\Gamma_{h} w_{h}\right)-G_{h}\left(u-u_{h}, v_{h}\right)+G_{h}\left(u, v_{h}\right) \\
= & \left(u-u_{h}, v-v_{h}\right)-d\left(v-v_{h}, p-p_{h}\right)+d\left(u-u_{h}, w-w_{h}\right) \\
& +\left(f, w_{h}-\Gamma_{h} w_{h}\right)-G_{h}\left(u-u_{h}, v_{h}\right)+G_{h}\left(u, v_{h}\right) .
\end{aligned}
$$

Combining (2.3), (5.3) with Theorem 2 yields

$$
\begin{aligned}
\left|\left(u-u_{h}, v-v_{h}\right)-d\left(v-v_{h}, p-p_{h}\right)+d\left(u-u_{h}, w-w_{h}\right)\right| \\
\leq C\left\|u-u_{h}\right\|_{0}\left\|v-v_{h}\right\|_{0}+\left\|v-v_{h}\right\|_{0}\left\|\nabla\left(p-p_{h}\right)\right\|_{0} \\
\quad+\left\|u-u_{h}\right\|_{0}\left\|\nabla\left(w-w_{h}\right)\right\|_{0} \\
\leq C h^{2}\left(\|u\|_{1}+\|p\|_{2}+\|f\|_{0}\right)\left(\|v\|_{1}+\|w\|_{2}\right) \\
\leq C h^{2}\left(\|u\|_{1}+\|p\|_{2}+\|f\|_{0}\right)\left\|Q_{\tau_{1}} \phi\right\|_{0} .
\end{aligned}
$$


By (2.5)-(2.7), it suffices to show that

$$
\begin{aligned}
& \left|-G_{h}\left(u-u_{h}, v_{h}\right)+G_{h}\left(u, v_{h}\right)\right| \\
& \quad \leq\left(\left\|u-u_{h}\right\|_{0}+\left\|u-\Pi_{h} u\right\|_{0}\right)\left\|v_{h}-\Pi_{h} v_{h}\right\|_{0} \\
& \quad \leq\left(\left\|u-u_{h}\right\|_{0}+\left\|u-\Pi_{h} u\right\|_{0}\right)\left(\left\|v-v_{h}\right\|_{0}+\left\|v-\Pi_{h} v\right\|_{0}+\left\|\Pi_{h} v-\Pi_{h} v_{h}\right\|_{0}\right) \\
& \quad \leq C h^{2}\left(\|u\|_{1}+\|p\|_{2}+\|f\|_{0}\right)\left\|Q_{\tau_{1}} \phi\right\|_{0} .
\end{aligned}
$$

Furthermore, from Lemma 2 and (2.6) that

$$
\begin{aligned}
\left|\left(f, w_{h}-\Gamma_{h} w_{h}\right)\right| & =\left|\left(f-P_{h} f, w_{h}-\Gamma_{h} w_{h}\right)\right| \\
& \leq\left\|f-P_{h} f\right\|_{0}\left\|w_{h}-\Gamma_{h} w_{h}\right\|_{0} \leq C h^{2}\|f\|_{1}\left\|Q_{\tau_{1}} \phi\right\|_{0} .
\end{aligned}
$$

Combining above inequalities with (5.6), we finished the proof.

Lemma 6. Assume $P_{\tau_{2}} \in H^{1}(\Omega)^{2}$ and $f \in H^{1}(\Omega)$, there is a constant $C$ independent of $h$ and $\tau_{2}$ such that

$$
\left\|R_{\tau_{2}} u-R_{\tau_{2}} u_{h}\right\|_{0} \leq C h^{2-\alpha_{2}}\left(\|p\|_{2}+\|u\|_{1}+\|f\|_{1}\right)
$$

where $\alpha_{2} \in(0,1)$ is defined in $(5.1)$.

Proof. From the definition of $\|\cdot\|_{0}$ and $R_{\tau_{2}}$, we have

$$
\begin{aligned}
& \left\|R_{\tau_{2}} u-R_{\tau_{2}} u_{h}\right\|_{0}=\sup _{\phi \in L^{2}(\Omega)^{2},\|\phi\|_{0}=1}\left|\left(R_{\tau_{2}} u-R_{\tau_{2}} u_{h}, \phi\right)\right|, \\
& \left(R_{\tau_{2}} u-R_{\tau_{2}} u_{h}, \phi\right)=\left(u-u_{h}, R_{\tau_{2}} \phi\right) .
\end{aligned}
$$

Then we obtain that

$$
\left\|R_{\tau_{2}} u-R_{\tau_{2}} u_{h}\right\|_{0}=\sup _{\phi \in L^{2}(\Omega)^{2},\|\phi\|_{0}=1}\left|\left(u-u_{h}, R_{\tau_{2}} \phi\right)\right| .
$$

Considering the following dual problem

$$
\left(u-u_{h}, v\right)+d\left(p-p_{h}, v\right)-d\left(u-u_{h}, w\right)=\left(u-u_{h}, R_{\tau_{2}} \phi\right) .
$$

Because of the convexity of domain $\Omega$, problem (5.7) has a unique solution and satisfies

$$
\|w\|_{2}+\|v\|_{1} \leq C\left\|R_{\tau_{2}} \phi\right\|_{1} .
$$

Combining (5.5) with (5.7), we obtain that

$$
\begin{aligned}
\left(u-u_{h}, R_{\tau_{2}} \phi\right)= & \left(u-u_{h}, v-v_{h}\right)+d\left(v-v_{h}, p-p_{h}\right)-d\left(u-u_{h}, w-w_{h}\right) \\
& +\left(\nabla \cdot\left(u-u_{h}\right), w_{h}-\Gamma_{h} w_{h}\right)-G_{h}\left(u-u_{h}, v_{h}\right)+G_{h}\left(u, v_{h}\right) \\
= & \left(u-u_{h}, v-v_{h}\right)+d\left(v-v_{h}, p-p_{h}\right)-d\left(u-u_{h}, w-w_{h}\right) \\
& +\left(f, w_{h}-\Gamma_{h} w_{h}\right)-G_{h}\left(u-u_{h}, v_{h}\right)+G_{h}\left(u, v_{h}\right) .
\end{aligned}
$$

Applying the tricks used in Lemma 5 and the inverse inequality, and combining (5.8), we completed the proof.

Now, we are ready to estimate $p-Q_{\tau_{1}} p_{h}$ and $u-R_{\tau_{2}} u_{h}$. 
Theorem 4. Under the assumptions of Lemma 5, if $\tau_{1}, h$ and $\alpha_{1}$ satisfy

$$
\tau_{1}=\mathcal{O}\left(h^{\alpha_{1}}\right) \quad \text { with } \alpha_{1}=2 /(r+1) \text {, }
$$

then the postprocessed solution $Q_{\tau_{1}} p_{h}$ satisfies

$$
\begin{gathered}
\left\|p-Q_{\tau_{1}} p_{h}\right\|_{0} \leq C h^{2}\left(\|p\|_{r+1}+\|p\|_{2}+\|u\|_{1}+\|f\|_{1}\right), \\
\left\|\nabla_{\tau_{1}}\left(p-Q_{\tau_{1}} p_{h}\right)\right\|_{0} \leq C h^{\frac{2 r}{1+r}}\left(\|p\|_{r+1}+\|p\|_{2}+\|u\|_{1}+\|f\|_{1}\right) .
\end{gathered}
$$

Proof. By the definition of $Q_{\tau_{1}}$, we have

$$
\begin{aligned}
\left\|p-Q_{\tau_{1}} p_{h}\right\|_{0} & \leq\left\|p-Q_{\tau_{1}} p\right\|_{0}+\left\|Q_{\tau_{1}} p-Q_{\tau_{1}} p_{h}\right\|_{0} \\
& \leq C \tau_{1}^{r+1}\|p\|_{r+1}+C h^{2}\left(\|p\|_{2}+\|u\|_{1}+\|f\|_{1}\right) \\
& \leq C h^{\alpha_{1}(r+1)}\|p\|_{r+1}+h^{2}\left(\|p\|_{2}+\|u\|_{1}+\|f\|_{1}\right) .
\end{aligned}
$$

The above error estimate can be optimized by choosing $\alpha_{1}$ such that

$$
\alpha_{1}(r+1)=2 \text {, }
$$

solving the above equation gives $\alpha_{1}=\frac{2}{r+1}$, we obtain the $L^{2}$-estimate of $p-Q_{\tau_{1}} p_{h}$. It is easy to see that

$$
\left\|\nabla_{\tau_{1}}\left(p-Q_{\tau_{1}} p\right)\right\|_{0} \leq C \tau_{1}^{r}\|p\|_{r+1}=C h^{\alpha_{1} r}\|p\|_{r+1},
$$

where $\nabla_{\tau_{1}}$ is defined elementwise over the partition $\mathcal{T}_{\tau_{1}}$. By the inverse inequality and Lemma 5 , we have

$$
\begin{aligned}
\left\|\nabla_{\tau_{1}}\left(p-Q_{\tau_{1}} p_{h}\right)\right\|_{0} & \leq\left\|\nabla_{\tau_{1}}\left(p-Q_{\tau_{1}} p\right)\right\|_{0}+\left\|\nabla_{\tau_{1}}\left(Q_{\tau_{1}} p-Q_{\tau_{1}} p_{h}\right)\right\|_{0} \\
& \leq C\left(h^{\alpha_{1} r}\|p\|_{r+1}+h^{2-\alpha_{1}}\left(\|p\|_{2}+\|u\|_{1}+\|f\|_{1}\right)\right) .
\end{aligned}
$$

We optimize the above estimate by choosing $\alpha_{1}$ such that

$$
\alpha_{1} r=2-\alpha_{1}
$$

then, we obtain the following estimate

$$
\left\|\nabla_{\tau_{1}}\left(p-Q_{\tau_{1}} p_{h}\right)\right\|_{0} \leq C h^{\frac{2 r}{1+r}}\left(\|p\|_{r+1}+\|p\|_{2}+\|u\|_{1}+\|f\|_{1}\right) .
$$

Combining (5.9) with (5.10), we finished the proof.

Similarly, we have the following result for velocity.

Theorem 5. Under the assumptions of Lemma 6, if $\tau_{2}, h$ and $\alpha_{2}$ satisfy

$$
\tau_{2}=\mathcal{O}\left(h^{\alpha_{2}}\right) \quad \text { with } \alpha_{2}=2 /(t+2) \text {, }
$$

then the postprocessed solution $R_{\tau_{2}} u_{h}$ satisfies

$$
\left\|u-R_{\tau_{2}} u_{h}\right\|_{0} \leq C h^{\frac{2(1+t)}{2+t}}\left(\|p\|_{2}+\|u\|_{t+1}+\|f\|_{1}\right) .
$$


Table 1. The results of standard finite volume method with $P_{1}$ element.

\begin{tabular}{lllll}
\hline $1 / h$ & $\frac{\left\|p_{h}-p\right\|_{0}}{\|p\|_{0}}$ & Order & $\frac{\left\|p_{h}-p\right\|_{1}}{\|p\|_{1}}$ & Order \\
\hline 10 & 0.1105 & $/$ & 0.3040 & $/$ \\
20 & 0.02891 & 1.9348 & 0.1551 & 0.9709 \\
30 & 0.01296 & 1.9788 & 0.1038 & 0.9905 \\
40 & 0.007310 & 1.9905 & 0.07792 & 0.9969 \\
50 & 0.004685 & 1.9937 & 0.06237 & 0.9976 \\
\hline
\end{tabular}

Proof. By the definition of $R_{\tau_{2}}$, we have

$$
\begin{aligned}
\left\|u-R_{\tau_{2}} u_{h}\right\|_{0} & \leq\left\|u-R_{\tau_{2}} u\right\|_{0}+\left\|R_{\tau_{2}} u-R_{\tau_{2}} u_{h}\right\|_{0} \\
& \leq C\left(\tau_{1}^{t+1}\|u\|_{t+1}+h^{2-\alpha_{2}}\left(\|p\|_{2}+\|u\|_{1}+\|f\|_{1}\right)\right) \\
& \leq C\left(h^{\alpha_{2}(t+1)}\|u\|_{t+1}+h^{2-\alpha_{2}}\left(\|p\|_{2}+\|u\|_{1}+\|f\|_{1}\right)\right) .
\end{aligned}
$$

The above error estimate can be optimized by choosing $\alpha_{2}$ such that

$$
\alpha_{2}(t+1)=2-\alpha_{2}
$$

Solving the above equation gives $\alpha_{2}=\frac{2}{t+2}$. With this chosen, combining (5.11) we completed the proof.

Finally, we have the following error estimates by choosing the different mesh sizes relationship.

Corollary 1. Assume that $V_{\tau_{1}} \in L^{2}(\Omega), P_{\tau_{2}} \in H^{1}(\Omega)^{2}$ and $f \in H^{1}(\Omega)$. If $\alpha_{1}=\alpha_{2}=\frac{2}{3}$, then we have

$$
\begin{aligned}
& \left\|p-Q_{\tau_{1}} p_{h}\right\|_{0} \leq C h^{2}\left(\|p\|_{3}+\|u\|_{1}+\|f\|_{1}\right), \\
& \left\|\nabla_{\tau_{1}}\left(p-Q_{\tau_{1}} p_{h}\right)\right\|_{0} \leq C h^{\frac{4}{3}}\left(\|p\|_{3}+\|u\|_{1}+\|f\|_{1}\right) \\
& \left\|u-R_{\tau_{2}} u_{h}\right\|_{0} \leq C h^{\frac{4}{3}}\left(\|p\|_{2}+\|u\|_{2}+\|f\|_{1}\right) .
\end{aligned}
$$

Besides, if $\alpha_{1}=\frac{2}{3}, \alpha_{2}=\frac{1}{2}$, then we arrive at

$$
\begin{aligned}
& \left\|p-Q_{\tau_{1}} p_{h}\right\|_{0} \leq C h^{2}\left(\|p\|_{3}+\|u\|_{1}+\|f\|_{1}\right), \\
& \left\|\nabla_{\tau_{1}}\left(p-Q_{\tau_{1}} p_{h}\right)\right\|_{0} \leq C h^{\frac{4}{3}}\left(\|p\|_{3}+\|u\|_{1}+\|f\|_{1}\right) \\
& \left\|u-R_{\tau_{2}} u_{h}\right\|_{0} \leq C h^{\frac{3}{2}}\left(\|p\|_{2}+\|u\|_{3}+\|f\|_{1}\right) .
\end{aligned}
$$

As a consequence, we can see that there is no improvement for the pressure in $L^{2}$-norm, but the superconvergence results for the gradient of pressure and velocity are established under some regularities of $p, u$ and $f$.

\section{$6 \quad$ Numerical Experiments}

In this section, we show some numerical examples to verify the established results in Sections 4 and 5. In all tests, we choose the domain $\Omega$ as the unit 
Table 2. The results of mixed finite volume method with $P_{1}^{2}-P_{1}$ element.

\begin{tabular}{cllllll}
\hline $1 / h$ & $\frac{\left\|p_{h}-p\right\|_{0}}{\|p\|_{0}}$ & Order & $\frac{\left\|p_{h}-p\right\|_{1}}{\|p\|_{1}}$ & Order & $\frac{\left\|u_{h}-u\right\|_{0}}{\|u\|_{0}}$ & Order \\
\hline 10 & 0.6450 & $/$ & 0.3439 & $/$ & 0.1752 & $/$ \\
20 & 0.1423 & 2.1804 & 0.1631 & 1.0762 & 0.08241 & 1.0881 \\
30 & 0.06186 & 2.0546 & 0.1079 & 1.0190 & 0.05437 & 1.0262 \\
40 & 0.03453 & 2.0267 & 0.08067 & 1.0110 & 0.04063 & 1.0128 \\
50 & 0.02202 & 2.0161 & 0.06445 & 1.0060 & 0.03245 & 1.0063 \\
\hline
\end{tabular}

Table 3. The superconvergence results with $P_{1}^{2}-P_{2}$ element with $\alpha_{1}=\alpha_{2}=\frac{2}{3}$.

\begin{tabular}{cllllll}
\hline $1 / h$ & $\frac{\left\|p_{h}-p\right\|_{0}}{\|p\|_{0}}$ & Order & $\frac{\left\|p_{h}-p\right\|_{1}}{\|p\|_{1}}$ & Order & $\frac{\left\|u_{h}-u\right\|_{0}}{\|u\|_{0}}$ & \multirow{2}{*}{ Order } \\
\hline 10 & 0.06621 & $/$ & 0.2586 & $/$ & 0.3882 & $/$ \\
20 & 0.01565 & 2.0809 & 0.08199 & 1.6572 & 0.1111 & 1.8049 \\
30 & 0.007034 & 1.9723 & 0.04768 & 1.3369 & 0.06383 & 1.3668 \\
40 & 0.003918 & 2.0341 & 0.03063 & 1.5383 & 0.04142 & 1.5033 \\
50 & 0.002444 & 2.1150 & 0.02178 & 1.5281 & 0.02910 & 1.5821 \\
\hline
\end{tabular}

Table 4. The superconvergence results with $P_{2}^{2}-P_{2}$ element with $\alpha_{1}=\frac{2}{3}, \alpha_{2}=\frac{1}{2}$.

\begin{tabular}{cllllll}
\hline $1 / h$ & $\frac{\left\|p_{h}-p\right\|_{0}}{\|p\|_{0}}$ & Order & $\frac{\left\|p_{h}-p\right\|_{1}}{\|p\|_{1}}$ & Order & $\frac{\left\|u_{h}-u\right\|_{0}}{\|u\|_{0}}$ & Order \\
\hline 10 & 0.07612 & $/$ & 0.24585 & $/$ & 0.06982 & $/$ \\
20 & 0.01679 & 2.1807 & 0.08506 & 1.5312 & 0.03062 & 1.1892 \\
30 & 0.007622 & 1.9478 & 0.05038 & 1.2918 & 0.01641 & 1.5384 \\
40 & 0.004063 & 2.1868 & 0.03212 & 1.5646 & 0.01065 & 1.5028 \\
50 & 0.002641 & 1.9304 & 0.02309 & 1.4792 & 0.007387 & 1.6395 \\
\hline
\end{tabular}

square $\Omega=[0,1] \times[0,1]$, the exact solution $p=\sin (2 \pi x) \sin (2 \pi y)$ and $f=$ $8 \pi^{2} \sin (2 \pi x) \sin (2 \pi y)$ is determined by $(2.1)$.

Firstly, we provide the numerical results of standard finite volume method for (2.1) with different meshes, which are shown in Table 1. And then, we give the results obtained by mixed finite volume method (3.1) in different meshes. Compared with the results in Table 1, we can see that there are no much differences of the errors between two methods in $H^{1}$-norm, but we can obtain the better results in $L^{2}$-norm by using mixed method. Furthermore, the results in Table 2 verify the established theoretical findings of Theorems 2-3.

Finally, in order to achieve superconvergence for the numerical solution, the local $L^{2}$ projections are used. The key of this method is to project one finite element space onto another one based on the high order polynomials of the coarse mesh. The solution of $\left(Q_{\tau_{1}} p_{h}, R_{\tau_{2}} u_{h}\right)$ can be computed as follows: Find $\left(Q_{\tau_{1}} p_{h}, R_{\tau_{2}} u_{h}\right) \in\left(V_{\tau_{1}}, P_{\tau_{2}}\right)$ for all $(w, v) \in\left(V_{\tau_{1}}, P_{\tau_{2}}\right)$ such that

$$
\left(Q_{\tau_{1}} p_{h}, w\right)=\left(p_{h}, w\right), \quad\left(R_{\tau_{2}} u_{h}, v\right)=\left(u_{h}, v\right),
$$

where $\left(p_{h}, u_{h}\right)$ is the solution obtained by (3.1). Tables $3-4$ list the mesh sizes among different partitions and the convergence rates of numerical solutions. 


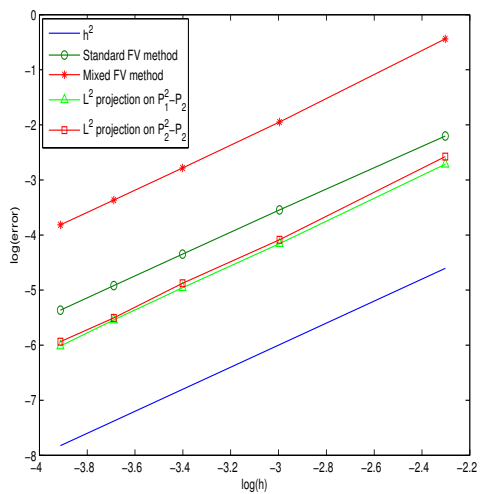

(a)

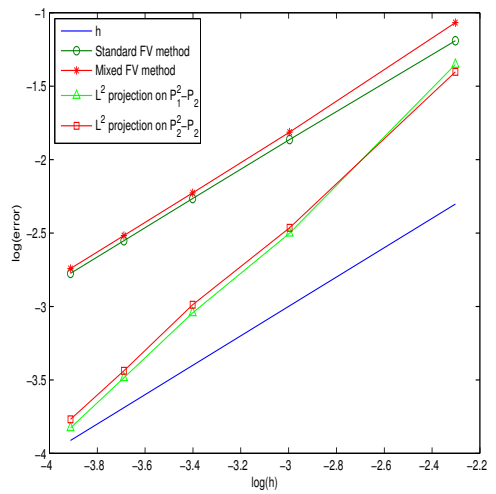

(b)

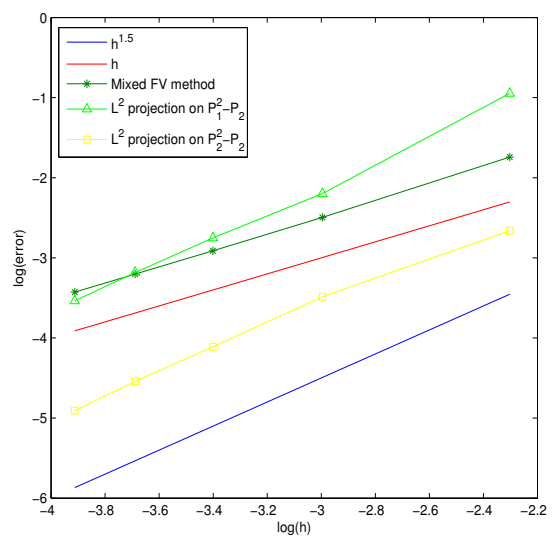

(c)

Figure 1. Errors of various variables of different methods for Poisson equations. (a) $L^{2}$-error for pressure, (b) $H^{1}$-error for the pressure, (c) $L^{2}$-error for velocity.

From those Tables, we can see that the relative errors of pressure obtained by $L^{2}$ projection method are better than these in Table 2, and the convergence orders confirm the results of Corollary 1, see also Figure 1 for details.

\section{Conclusions}

In this paper we have presented a theoretical analysis of stabilized finite volume method for Poisson equation. By introducing a new mixed variational formulation, we obtained the optimal error estimates for the numerical solutions based on the linear polynomials over the triangulation partitions. Furthermore, with the help of the $L^{2}$-projection method, we presented some superconvergence results for the numerical solutions under some regularity assumptions for Poisson equation. Some numerical experiments are presented at last. 


\section{Acknowledgments}

The authors sincerely thank the editor and anonymous reviewers for their valuable comments and suggestions which led to a large improvements of the paper.

\section{References}

[1] R. Bank and D. Rose. Some error estimates for the box method. SIAM J. Numer. Anal., 24(4):777-787, 1987. http://dx.doi.org/10.1137/0724050.

[2] P. Bochev, C. Dohrmann and M. Gunzburger. Stabilization of low-order mixed finite elements for the Stokes equations. SIAM J. Numer. Anal., 44:82-101, 2006. http://dx.doi.org/10.1137/S0036142905444482.

[3] S. Brenner and L. Scott. The Mathematical Theory of Finite Element Methods. Springer-Verlag, 1994.

[4] Z.X. Chen. Finite Element Methods and Their Application. Springer-Verlag, Heidelberg, 2005.

[5] S.H. Chou and X. Ye. Super convergence of finite volume methods for the second order elliptic problem. Comput. Methods Appl. Mech. Engrg., 196:3706-3712, 2007. http://dx.doi.org/10.1016/j.cma.2006.10.025.

[6] M. Cui and X. Ye. Super convergence of finite volume methods for the Stokes equations. Numer. Methods Partial Differential Equations, 25:1212-1230, 2009. http://dx.doi.org/10.1002/num.20399.

[7] R. Ewing, T. Lin and Y.P. Lin. On the accuracy of the finite volume element based on piecewise linear polynomials. SIAM J. Numer. Anal., 39(6):1865-1888, 2002. http://dx.doi.org/10.1137/S0036142900368873.

[8] R. Eymard, T. Gallouet and R. Herbin. Finite volume methods. In P.G. Ciarlet and J.L. Lions(Eds.), Handbook of Numer. Anal., volume 2, pp. 713-1020, 1997.

[9] Y.N. He. A fully discrete stabilized finite element method for the timedependent Navier-Stokes problem. IMA J. Numer. Anal., 23:665-691, 2003. http://dx.doi.org/10.1093/imanum/23.4.665.

[10] J. Li and Z.X. Chen. A new stabilized finite volume method for the stationary Stokes equations. Adv. Comput. Math., 30:141-152, 2009.

http://dx.doi.org/10.1007/s10444-007-9060-5.

[11] J. Li and Z.X. Chen. On the semi-discrete stabilized finite volume method for the transient navier-stokes equations. Adv. Comput. Math., 38(2):281-320, 2013. http://dx.doi.org/10.1007/s10444-011-9237-9.

[12] J. Li and Y.N. He. A stabilized finite element method based on two local Gauss integrations for the stokes equations. J. Comput. Appl. Math., 214:58-65, 2008. http://dx.doi.org/10.1016/j.cam.2007.02.015.

[13] J. Li, Y.N. He and Z.X. Chen. A new stabilized finite element method for the transient Navier-Stokes equations. Comput. Methods Appl. Mech. Engng., 197:22-35, 2007. http://dx.doi.org/10.1016/j.cma.2007.06.029.

[14] J. Li, L.H. Shen and Z.X. Chen. Convergence and stability of a stabilized finite volume method for the stationary Navier-Stokes equations. BIT Numer. Math., 50:823-842, 2010. http://dx.doi.org/10.1007/s10543-010-0277-1. 
[15] J. Li, J.P. Wang and X. Ye. Super convergence by $l^{2}$ projections for stabilized finite element methods for the stokes equations. Int. J. Numer. Anal. Model., 6:711-723, 2009.

[16] J. Li, X. Zhao, J.H. Wu and J.H. Yang. A stabilized low order finite-volume method for the three dimensional stationary Navier-Stokes equations. Math. Prob. Engng., 2012:1-14, 2012. http://dx.doi.org/10.1155/2012/297269.

[17] R.H. Li and P. Zhu. Generalized difference methods for second order elliptic partial differential equations(I)-triangle grids. Numer. Math. J. Chinese Univ., 2:140-152, 1982.

[18] F. Shi, J.P. Yu and K.T. Li. A new stabilized mixed finite element method for Poisson equation based on two local Gauss integrations for linear element pair. Int. J. Comput. Math., 88:2293-2305, 2011. http://dx.doi.org/10.1080/00207160.2010.534466.

[19] R. Temam. Navier-Stokes Equation: Theory and Numerical Analysis. NorthHolland, Amsterdam, New York, Oxford, 1984.

[20] J.P. Wang. A superconvergence analysis for finite element solutions by the leastsquares surface fitting on irregular meshes for smooth problems. J. Math. Study, 33:229-243, 2000.

[21] H.J. Wu and R.H. Li. Error estimates for finite volume element methods for general second-order elliptic problems. Numer. Methods Partial Differential Equations, 19:693-708, 2003. http://dx.doi.org/10.1002/num.10068.

$[22] \mathrm{X}$. Ye. On the relationship between finite volume and finite element methods applied to the stokes equations. Numer. Methods Partial Differential Equations, 17:440-453, 2001. http://dx.doi.org/10.1002/num.1021.

[23] H. Zhong. Numerical Research for the Stabilized Finite Volume Element Methods. Xi'an Jiaotong University, Master thesis, 2007. 